\title{
Vitexin suppresses renal cell carcinoma by regulating mTOR pathways
}

\author{
Yuhong Li ${ }^{1 \#}$, Qinghai $\mathrm{Sun}^{2 \#}$, Hui $\mathrm{Li}^{3}$, Bin Yang ${ }^{4}$, Meng Wang ${ }^{3 \wedge}$ \\ ${ }^{1}$ Department of Pharmacy, The First People's Hospital of Jingmen, Jingmen, China; ${ }^{2}$ Clinical Medicine Discipline, Weifang Traditional Chinese \\ Hospital, Weifang, China; ${ }^{3}$ Department of Medicine, ${ }^{4}$ Department of Vascular Surgery, Jining No. 1 People's Hospital, Jining, China \\ Contributions: (I) Conception and design: Y Li, Q Sun; (II) Administrative support: M Wang; (III) Provision of study materials or patients: H Li, B \\ Yang; (IV) Collection and assembly of data: Y Li, Q Sun; (V) Data analysis and interpretation: Y Li, Q Sun; (VI) Manuscript writing: All authors; (VII) \\ Final approval of manuscript: All authors. \\ "These authors contributed equally to this work. \\ Correspondence to: Meng Wang. Department of Medicine, Jining No. 1 People's Hospital, No. 6 Jiankang Road, Rencheng District, Jining 272000, \\ China. Email: auht0fk5sa@sina.com.
}

Background: Renal cell carcinoma (RCC) is one of the most common malignant tumors in the world. Vitexin (apigenin-8-C-D-glucopyranoside), a bioactive compound isolated from a variety of plants, has multiple protective effects on human health. The purpose of this study was to investigate the role of vitexin in $\mathrm{RC}$ and the related molecular mechanism.

Methods: Proliferation was tested with Cell Counting Kit- 8 and Edu staining. Apoptosis was studied with flow cytometry. Immunofluorescent was applied to show LC3 spots. BALB/c nude mice bearing ACHN cells were established and immunohistochemical staining was applied to validate the in vivo effects of vitexin. All the effects and possible signaling pathways involved were validated with western blotting.

Results: Seventy micromole of vitexin started to show significant effect on the growth of normal renal tubular epithelial cells (HK-2), so $0,10,20$ and $40 \mu M$ of vitexin were used in later experiments. Vitexin inhibited growth and induced apoptosis of ACHN and OS-RC-2 cells in a dose-dependent manner, and promoted excessive autophagy by reducing p62 levels and increasing Beclin1 and LC3II levels. Western blotting revealed that vitexin significantly increased the phosphorylation levels of Adenosine Monophosphate Activated Protein Kinase (AMPK) and c-Jun N-terminal kinase (JNK) in ACHN and OS-RC-2 cells, while decreasing the phosphorylation levels of phosphatidylinositol 3-kinase/activates protein kinase/mammalian target of rapamycin (PI3K/AKT/mTOR). In BALB/c nude mice bearing ACHN cells, vitexin inhibited tumor growth, reduced Ki67 and increased caspase-3 levels in the tumor tissues.

Conclusions: The results indicated that the tumor suppressive role of vitexin in ACHN and OSRC-2 cells involved AMPK/mTOR, PI3K/AKT/mTOR, and JNK pathways. Therefore, vitexin may be a promising drug for the treatment of RCC.

Keywords: Renal cell carcinoma (RCC); vitexin; autophagy; phosphatidylinositol 3-kinase/activates protein kinase/mammalian target of rapamycin (PI3K/AKT/mTOR); AMPK/mTOR; c-Jun N-terminal kinase (JNK)

Submitted Jun 10, 2020. Accepted for publication Jul 29, 2020.

doi: $10.21037 /$ tau-20-1094

View this article at: http://dx.doi.org/10.21037/tau-20-1094

^ ORCID: 0000-0002-9929-2669. 


\section{Introduction}

Renal cell carcinoma (RCC) is a common malignant tumor of the urinary system. An estimated 330,000 patients worldwide are diagnosed with RCC each year, and 140,000 patients die of this disease (1). The main treatment for RCC is a combination of surgery, conventional radiotherapy, and chemotherapy, but the results are inadequate (2), and distant metastases cause the condition of RC patient as to deteriorate. Targeted therapy has improved the patient survival rate, but due to high levels of drug resistance in the tumor cells, development is limited. Therefore, it is still necessary to find effective drugs with minimal side effects in the treatment of RCC.

Vitexin (apigenin-8-CD-glucopyranoside) is a bioactive flavonoid isolated from millet, fenugreek, and other traditional plants (3). Multiple studies have shown that vitexin has antioxidant (4), anticancer (5), anti-inflammatory (6), and antidiabetic effects (7). Vitexin extracted from Colocasia esculenta Linn leaves has shown weak diuretic activity in hypertensive rats with renal artery occlusion (8). However, the effect of vitexin on RCC and its related molecular mechanism have not been established.

Autophagy plays an important role in cell proliferation and apoptosis, and autophagy dysfunction can lead to the accumulation of damaged proteins and the damage of developmental genes. Autophagy is involved in the development of tumors such as skin cancer, gastric cancer, prostate cancer, and RCC (9). Vitexin 6 enhances autophagy and induces apoptosis in breast, colon, lung, bladder, and leukemia cells by activating the c-Jun $\mathrm{N}$-terminal kinase (JNK) pathway (10). However, whether vitexin can regulate the occurrence of RCC through autophagy is still unknown.

Mammalian target of rapamycin (mTOR) is a serine/ threonine kinase involved in cell proliferation. The mTOR pathway is mainly regulated by phosphatidylinositol 3-kinase/RAC-alpha serine/threonine-protein kinase (PI3K/AKT) (11) and AMPK (12). PI3K/AKT can induce the activation of mTOR Complex 1 (mTORC1), which has a negative regulatory effect on apoptosis (13). Studies report that PI3K/AKT/mTOR plays an important role in apoptosis and autophagy of human prostate cancer and renal cells (14) and that autophagy is regulated by the AMPK/ mTOR pathway which is involved in renal ischemiareperfusion injury and RCC (12).

To date, the molecular mechanism of vitexin and its role in RCC are not clear. In this study, we discuss the role of vitexin in RCC, and study its possible mechanism. Our results suggest that vitexin may be a promising drug for RCC treatment.

We present the following article in accordance with the ARRIVE reporting checklist (available at http://dx.doi. org/10.21037/tau-20-1094).

\section{Methods}

\section{Cell culture and treatment}

Human RCC cell lines OS-RC-2 and ACHN, and human normal tubular epithelial cell line HK-2, were purchased from the Chinese Academy of Sciences (Shanghai, China). ACHN and HK-2, OS-RC-2 cells were cultured in Dulbecco's Modified Eagle's Medium (DMEM; Gibco; Thermo Fisher Scientific, Inc., Waltham, MA, USA), DMEM and RPMI-1640 (Gibco; Thermo Fisher Scientific, Inc., Waltham, MA, USA). HK-2 culture medium was added with $5 \mathrm{mM}$ glucose. All media were supplemented with $10 \%$ fetal calf serum (FBS; Gibco; Thermo Fisher Scientific, Inc., Waltham, MA, USA) and 1\% penicillin/ streptomycin (HyClone; GE Healthcare Life Sciences, Logan, UT, USA). Vitexin (CAS: 3681-93-4) was purchased from Sigma (St. Louis, MO, USA). The ACHN and OSRC-2 cells were divided into the four following groups: a control group, with cells treated with physiological saline; vitexin groups $(10,20,40 \mu \mathrm{M})$, with cells treated with vitexin $(10,20,40 \mu M)$ for $24 \mathrm{~h}(15)$.

\section{CCK-8 assay}

Vitexin-induced cytotoxicity was tested with a CCK-8 cell counting kit (Cell Counting Kit-8, Dojindo, Japan) as previously reported (16). HK-2 cells were cultured in 96well plates and treated with vitexin $(0,10,20,30,40,50$, $60,70,80,90$, and $100 \mu \mathrm{M})$ for $24 \mathrm{~h}$. The plates were then incubated for $22 \mathrm{~h}$ at $37{ }^{\circ} \mathrm{C}$ with $5 \% \mathrm{CO}_{2}$. After $10 \mu \mathrm{L}$ of CCK-8 solution was added to each well and cultured for more $2 \mathrm{~h}$. The absorbance at $450 \mathrm{~nm}$ was examined in each well by a multi-function microplate reader SpectraMax M5 (Molecular Devices, Sunnyvale, CA, USA).

\section{Edu staining}

The cells $\left(1 \times 10^{6}\right)$ were inoculated into a culture flask $\left(25 \mathrm{~cm}^{2}\right)$. The Click-iTTM 5-ethynyl-2'-deoxyuridine (EdU, Molecular Probes; Invitrogen) solvent was diluted to $20 \mu \mathrm{M}$ with the corresponding medium. The diluted Edu solvent 
was added to the flask for $24 \mathrm{~h}$. Then, the cells were fixed with $4 \%$ paraformaldehyde for $15 \mathrm{~min}$, stained with Alexa Fluor 594 overnight, cell nuclei were counterstained with DAPI for $20 \mathrm{~min}$, and observed by a fluorescence microscope (Eclipse 80i, Nikon). The results were analyzed using Image-Pro Plus 6.0 software (Media Cybernetics, Inc., USA).

\section{Western blotting}

After washing with cold phosphate-buffered saline (PBS), the cells were lysed in ice-cold RIPA (RadioImmunoprecipitation Assay) Buffe (Sigma-Aldrich; Merck KGaA) containing a phosphatase inhibitor. The total amount of protein was monitored by Bicinchoninic Acid (BCA) Protein Assay Kit (Beyotime Institute of Biotechnology, Haimen, China). About $30 \mu \mathrm{g}$ protein of each sample was used in electrophoresis. After separation on sodium dodecyl sulfate-polyacrylamide gel electrophoresis (SDS-PAGE) gels, the protein was transferred onto a polyvinylidene fluoride (PVDF) membrane (Millipore, IPFL00010, Billerica, MA, USA) and probed with primary antibodies: Ki-67 (sc-23900, 1:1,000, Santa Cruz Biotechnology, USA), PCNA (\#2586, 1:2,000, Cell Signaling Technology), Caspase-3 (\#9668, 1:1,000, CST, USA), Cleaved Caspase-3 (\#9661, 1:1,000, CST, USA), Caspase-9 (\#9508, 1:1,000, CST, USA), Cleaved Caspase-9 (\#20750, 1:1,000, CST, USA), p62 (\#88588, 1:1,000, CST, USA), Beclin 1 (\#4122, 1:1,000, CST, USA), LC3I (\#4599, 1:1,000, CST, USA), LC3II (\#3868, 1:1,000, CST, USA), AMPK $\alpha 1$ (\#2795, 1:1000, CST, USA), p-AMPK $\alpha 1$ (\#2795, 1:1,000, CST, USA), PI3K (\#4255, 1:1,000, CST, USA), p-PI3K (\#4228, 1:1,000, CST, USA), AKT (\#2920, 1:2,000, CST, USA), p-AKT (\#4060, 1:2,000, CST, USA), mTOR (\#4517, 1:1,000, CST, USA), p-mTOR (\#5536, 1:1,000, CST, USA), JNK (\#9252, 1:1,000, CST, USA), p-JNK (\#4668, 1:1,000, CST, USA), and GAPDH (\#97166, 1:1,000, CST, USA). After incubation with the primary antibodies, the membrane was incubated with the corresponding secondary antibodies and monitored with an enhanced chemiluminescence (ECL) substrate kit (Amersham Biosciences). Protein bands were photographed using an Odyssey two-color infrared laser imaging system (LI-COR Biosciences).

\section{Flow cytometry analysis of apoptosis rate and immune cells}

The apoptosis rate was detected by flow cytometry (Becton
Dickinson, San Jose, CA, USA) using Annexin V-fluorescein isothiocyanate (FITC) apoptosis detection kit (KeyGEN Biotech, Jiangsu, China) according to previous research (17). Annexin V-FITC (-)/Propidium Iodide (PI) (-) (the lower left) are normal cells, Annexin V-FITC (+)/PI (-) cells (the lower right) are early apoptotic cells, Annexin V-FITC (+)/ PI (+) (the upper right) are late apoptosis cells, and Annexin $\mathrm{V}(-) / \mathrm{PI}(+)$ (the upper left) are necrotic cells. For immune cells, anti-mouse CD3-eFluor450 (eBioscience, CA, USA) was added to the peripheral blood and incubated for $30 \mathrm{~min}$ at room temperature in the dark. Then, the erythrocyte lysis solution was added to the sample and incubated for another $20 \mathrm{~min}$. The cells were washed and re-suspended with PBS, and then analyzed by Beckman Gallios flow cytometer (Beckman Coulter, Inc., CA, USA).

\section{Immunofluorescent assay}

LC3 levels in ACHN cells and OS-RC-2 cells were detected by immunofluorescence assay. Cells were exposed to vitexin $(10,20$, and $40 \mu \mathrm{M})$ for $24 \mathrm{~h}$. Then, the cells were fixed with $4 \%$ paraformaldehyde for $30 \mathrm{~min}$, infiltrated with $0.5 \%$ Triton X-100 for another $30 \mathrm{~min}$, and blocked with tris-buffered saline with Tween20 (TBST) containing $5 \%$ bovine serum albumin (BSA, Affymetrix, Cleveland, $\mathrm{OH}$, USA) overnight at $37^{\circ} \mathrm{C}$. The sample was hybridized with the primary antibody LC3 (\#4108, 1:200, CST, USA) coupled with AlexaFluor ${ }^{\circledR} 647$ Conjugate secondary antibody overnight at $4{ }^{\circ} \mathrm{C}(\# 4414,1: 2,000$, CST, USA) and stained with DAPI (1:1,000, catalog number D9564, SigmaAldrich) at room temperature for $3 \mathrm{~h}$. The cells are then washed with PBS and observed by confocal microscopy (LSM 510 Meta, Zeiss, Oberkochen, Germany). LC3 spots were calculated from more than 10 microscopic fields.

\section{Tumor xenograft mouse models}

All animal experiments were performed in accordance with the National Institutes of Health (NIH) Guide for the Care and Use of Laboratory Animals and were approved by Guizhou Medical University. Forty BALB/c nude mice (5 mice per cage, six weeks old, 16-17 g body weight) were purchased from the Animal Center of Guizhou Medical University (Guiyang, China). Mice were housed in controlled conditions at $25 \pm 2{ }^{\circ} \mathrm{C}, 70 \%$ humidity, and a 12-h light-dark cycle, with regular sterile chow diet and water. Then, $3.2 \times 10^{6}$ ACHN cells were subcutaneously inoculated into the right flank of the mice. After successful 
modeling, the tumor-bearing mice were divided into four groups $(\mathrm{n}=10)$ and intraperitoneal injected daily with: saline (Control); vitexin $1 \mathrm{mg} / \mathrm{kg} / \mathrm{d}$; vitexin $2 \mathrm{mg} / \mathrm{kg} / \mathrm{d}$; and vitexin $4 \mathrm{mg} / \mathrm{kg} / \mathrm{d}$. After treatment for 25 days, the mice were sacrificed and the blood and formative tumors were collected for subsequent experiments.

The fixed tumor tissues were paraffin embedded to generate 5 - $\mu \mathrm{m}$ sections. Then the rehydration sections were stained with Ki67 and Caspase-3 primary antibodies respectively. The sections were then incubated with secondary antibodies (ABC kit, Vector Laboratories. Burlingame, CA, USA) for $1 \mathrm{~h}$ at room temperature. The staining was observed with a fluorescence microscope (Olympus, Tokyo, Japan) and analyzed with Image Pro Plus (version 6.0, Media Cybernetics).

\section{Statistical analysis}

Statistical analysis was performed with SPSS 22.0 (SPSS, Inc., Chicago, IL, USA). Measurement data are presented as mean \pm standard deviation (SD) $(\bar{x} \pm S)$ analyzed by $t$-test or one-way analysis of variance (ANOVA). $\mathrm{P}<0.05$ was considered statistically significant.

\section{Results}

Vitexin inbibited the growth of ACHN and OS-RC-2 cells

CCK-8 assay, EDU staining, and flow cytometry assay were used to detect the cell growth and side effects of vitexin. Low concentrations of (less than $60 \mu \mathrm{M}$ ) vitexin had no significant effect on HK-2 cells (Figure 1A). The positive rates of EDU in ACHN and OS-RC-2 cells treated with vitexin were significantly and dose-dependently lower than those in the control group (Figure $1 B$ ). Western blotting analysis (Figure 1C,D) showed that the expression of proliferation marker proteins $\mathrm{Ki}-67$ and PCNA were significantly inhibited by vitexin in ACHN and OS-RC-2 cells (Figure 1E,F). This indicated that low concentrations of vitexin inhibited the growth of ACHN and OS-RC-2 cells.

\section{Vitexin induced apoptosis of ACHN and OS-RC-2 cells}

The flow cytometry indicated apoptosis rates of ACHN and OS-RC-2 cells were increased in a dose-dependent manner after treatment with vitexin $(10,20$, and $40 \mu M)$
(Figure $2 A, B$ ). In addition, the expression of the apoptotic marker proteins Caspase-3, Caspase-9, Cleaved Caspase-3, and Cleaved Caspase-9 were promoted in a dose-dependent manner after treatment with vitexin $(10,20$, and $40 \mu \mathrm{M})$ (Figure 2C), thereby accelerating the apoptosis of ACHN and OS-RC-2 cells. The corresponding protein levels are shown in Figure 2D,E.

\section{Vitexin induced hyperautophagy of ACHN and OS-RC-2 cell}

Western blotting and immunofluorescence assay were used to detect the expression of autophagy-related proteins. Immunofluorescence assay showed that LC3 positive spots of ACHN and OS-RC-2 cells were increased in a dosedependent manner after treatment with vitexin $(10,20$ and $40 \mu \mathrm{M})$ (Figure $3 A, B)$. In addition, Western blotting indicated that vitexin promoted the expression of Beclin1 and the conversion of LC3-I to LC3-II, and increased the degradation of p62 (Figure 3C). The corresponding protein levels are shown in Figure 3D,E,F. In summary, these results demonstrated that vitexin induced hyperautophagy in ACHN and OS-RC-2 cells.

\section{Vitexin dose-dependently regulated AMPK/mTOR and PI3K/AKT/mTOR pathways in ACHN and OS-RC-2 cells}

The molecular mechanism of vitexin effect on RCC was studied by Western blotting (Figure 4A). Phosphorylation levels of AMPK $\alpha 1$ and JNK were significantly increased in ACHN and OS-RC-2 cells treated with vitexin (10, 20, and $40 \mu \mathrm{M})$, while phosphorylation levels of PI3K, AKT, and mTOR were significantly reduced. The relevant protein levels are shown in Figure 4B,C,D,E,F. Together, these results suggest that vitexin dose-dependently regulates AMPK/mTOR, PI3K/AKT/mTOR and JNK pathways in ACHN and OS-RC-2 cells.

Vitexin inhibited the growth of OS-RC-2 cells and induced apoptosis and hyperautophagy through AMPK/mTOR, PI3K/AKT/mTOR, and FNK pathways

To further confirm the molecular mechanism of vitexin on RCC, molecular activators or inhibitors were used. Vitexin $(40 \mu \mathrm{M})$, AMPK inhibitor compound C (CC, 20 $\mu \mathrm{M})$ and metformin $(2 \mathrm{mM})$ were added to OS-RC-2 cell culture medium. The phosphorylation level of AMPK was 
A

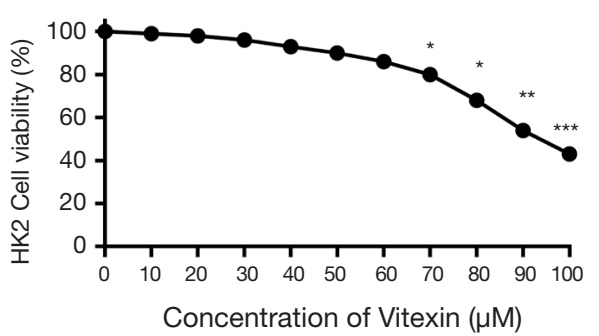

B

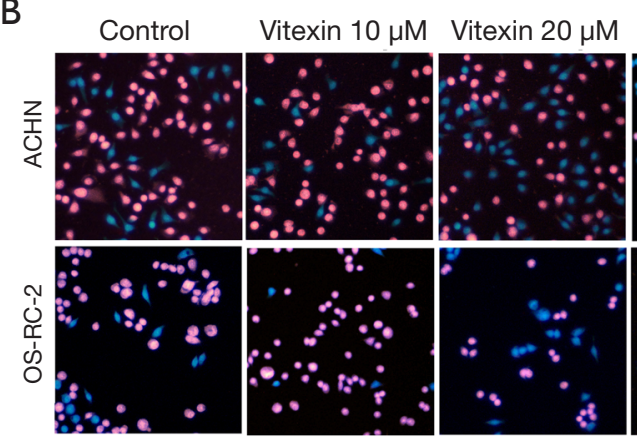

$\mathrm{C}$

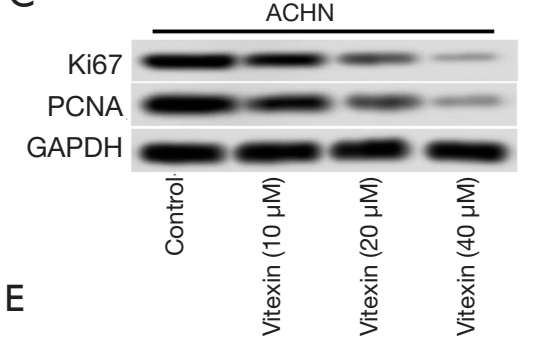

$\mathrm{D}$
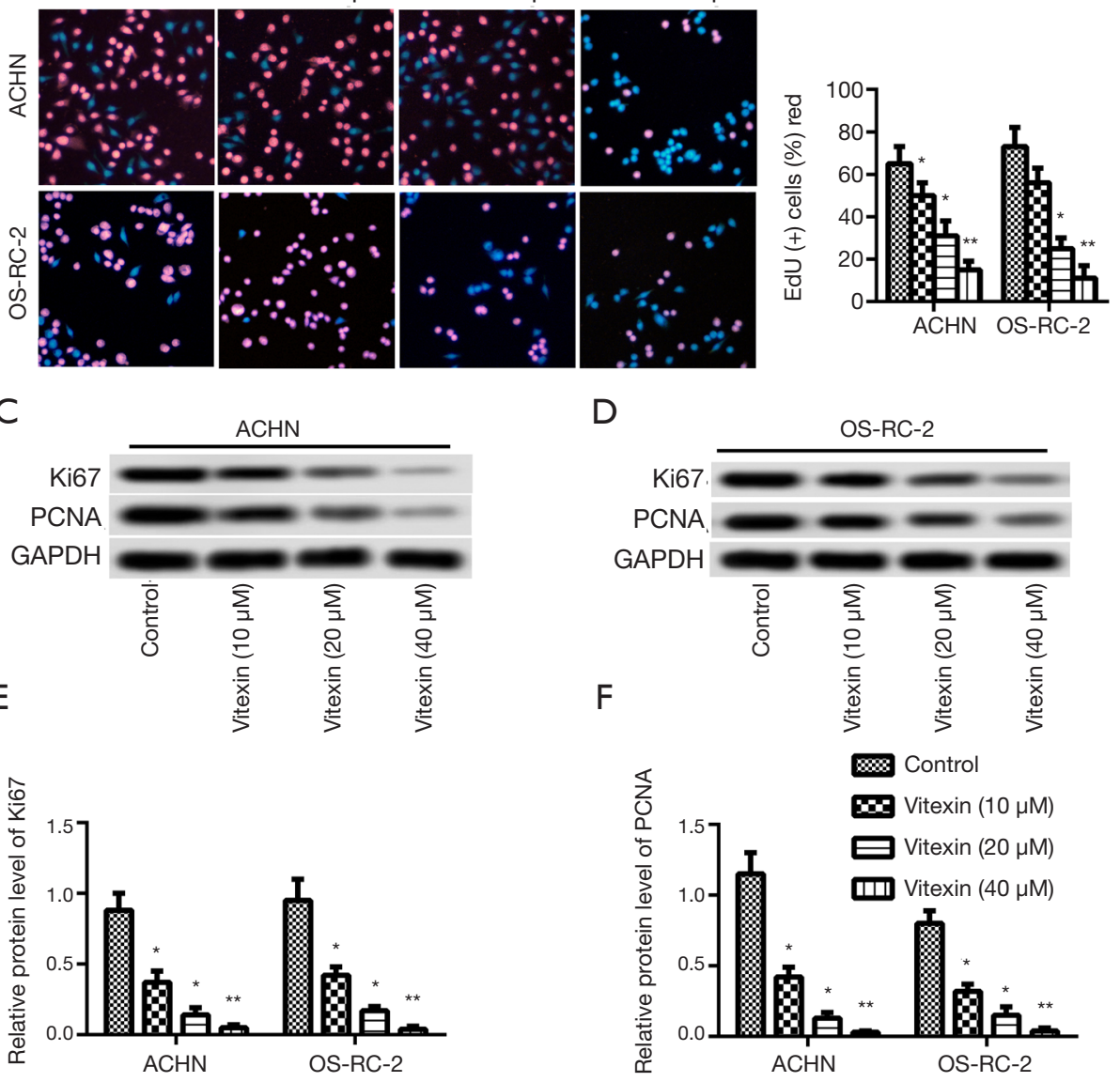

Figure 1 Vitexin inhibits the growth of ACHN and OS-RC-2 cells. (A) HK-2 cells were treated with doses of vitexin for $24 \mathrm{~h}(0,10,20,30$, 40, 50, 60, 70, 80, 90, and $100 \mu \mathrm{M}$ ). Cytotoxic effects of vitexin were measured by CCK-8 assay. (B) Cell growth of ACHN and OS-RC-2 cells was monitored by EdU staining observed under 100x scope. Proliferation of ACHN (C) and OS-RC-2 (D) cells were validated with relative protein expression of Ki67 (E) and PCNA (F). ( ${ }^{*}, \mathrm{P}<0.05$ vs. control; **, $\mathrm{P}<0.01$ vs. control; ***, $\left.\mathrm{P}<0.001\right)$.

significantly increased in the vitexin or metformin group and decreasing in the CC group (Figure $5 A$ ), indicating that vitexin, like metformin, promoted the phosphorylation of AMPK. In addition, after the addition of vitexin, CC, and rapamycin $(0.1 \mathrm{nM})$ to the OS-RC-2 cell culture medium, the phosphorylation level of mTOR also changed significantly (Figure 5B). The phosphorylation level of mTOR significantly decreased in the vitexin and rapamycin group, and increased in the CC group, indicating that vitexin, similar to rapamycin, inhibited the phosphorylation 
A

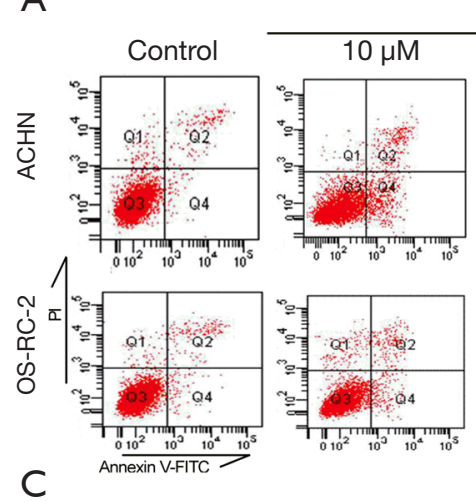

Caspase-3

c-Caspase-3

Caspase-9

c-Caspase-9

GAPDH

Caspase-3

c-Caspase-3

Caspase-9

c-Caspase-9

GAPDH
Vitexin
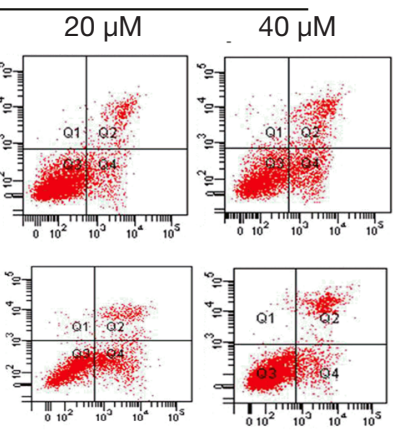

D

B

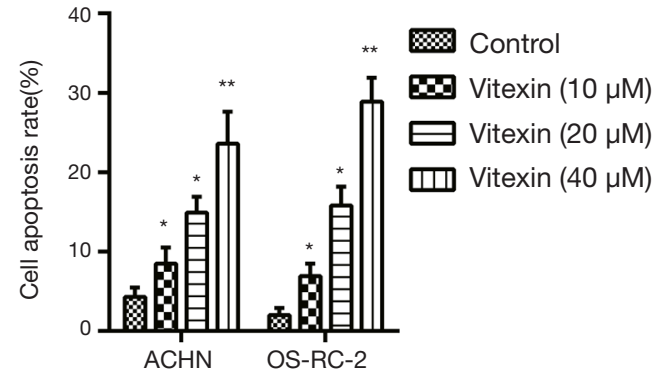

E
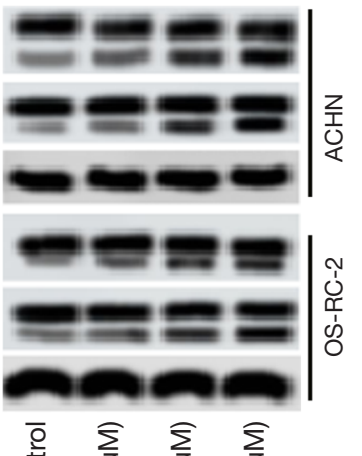

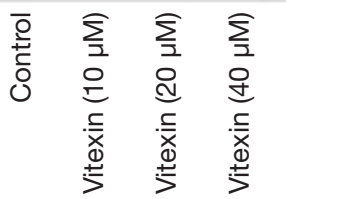
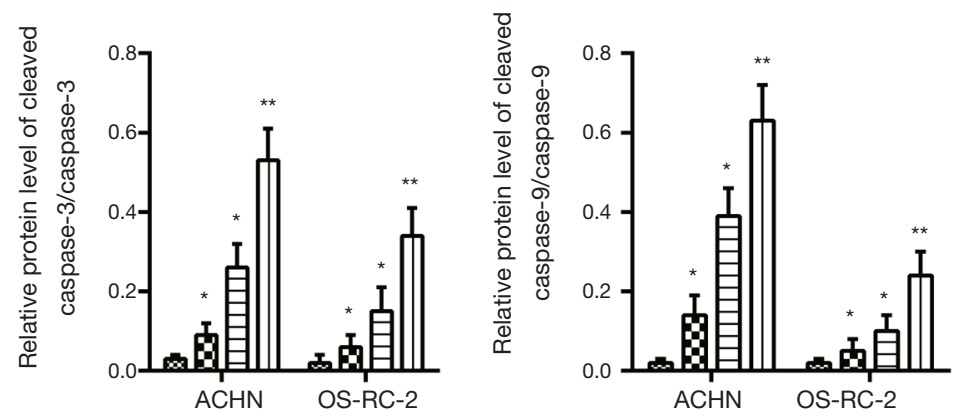

Figure 2 Vitexin induces apoptosis of ACHN and OS-RC-2 cells. ACHN and OS-RC-2 cells were treated with vitexin (0, 10, 20, 40 $\mu$ M) for 24 h. (A,B) Cell apoptosis assessed monitored by flow cytometry. (C) Protein levels of Caspase-3, Cleaved Caspase-3, Caspase-9, and Cleaved Caspase-9 were revealed by Western blotting. The ratio of cleaved Caspase-3/Caspase-3 (D) and cleaved Caspase-9/Caspase-9 (E) were calculated with Image J. GAPDH was used as inter reference $\left({ }^{*}, \mathrm{P}<0.05\right.$ vs. control; ${ }^{* *}, \mathrm{P}<0.01$ vs. control).

of mTOR. Furthermore, the phosphorylation levels of PI3K and AKT were significantly changed after vitexin and PI3K/AKT activator IGF-1 $(50 \mu \mathrm{M})$ were added to OS-RC-2 cell culture medium (Figure 5C). The addition of vitexin significantly inhibited the phosphorylation of PI3K and AKT. However, the addition of IGF-1 showed the opposite result. Finally, in the OS-RC-2 cell culture medium, vitexin and JNK inhibitor SP600125 (40 nM) were added separately or simultaneously, and the phosphorylation level of JNK changed significantly. As shown in Figure 5D, the phosphorylation level of JNK was significantly increased in the vitexin group and decreased in the SP600125 group, indicating that vitexin promoted the phosphorylation of JNK and induced autophagy. Taken together, these results confirm that vitexin inhibits the growth of OS-RC-2 cells and induces apoptosis and excessive autophagy by regulating AMPK/mTOR, PI3K/AKT/mTOR, and JNK pathways.

\section{Vitexin inbibited tumor formation in vivo}

To establish the effects of vitexin in vivo, BALB/c nude mice were incilated with ACHN cells. As shown in Figure $6 \mathrm{~A}$, vitexin treatment significantly reduced the weight of tumors in mice compared with the control group. No obvious adverse effects were observed. Flow cytometry analysis also showed that vitexin had no significant toxic effects on immune cells (Figure 6B). Immunohistochemistry results indicated that vitexin treatment also inhibited the expression 

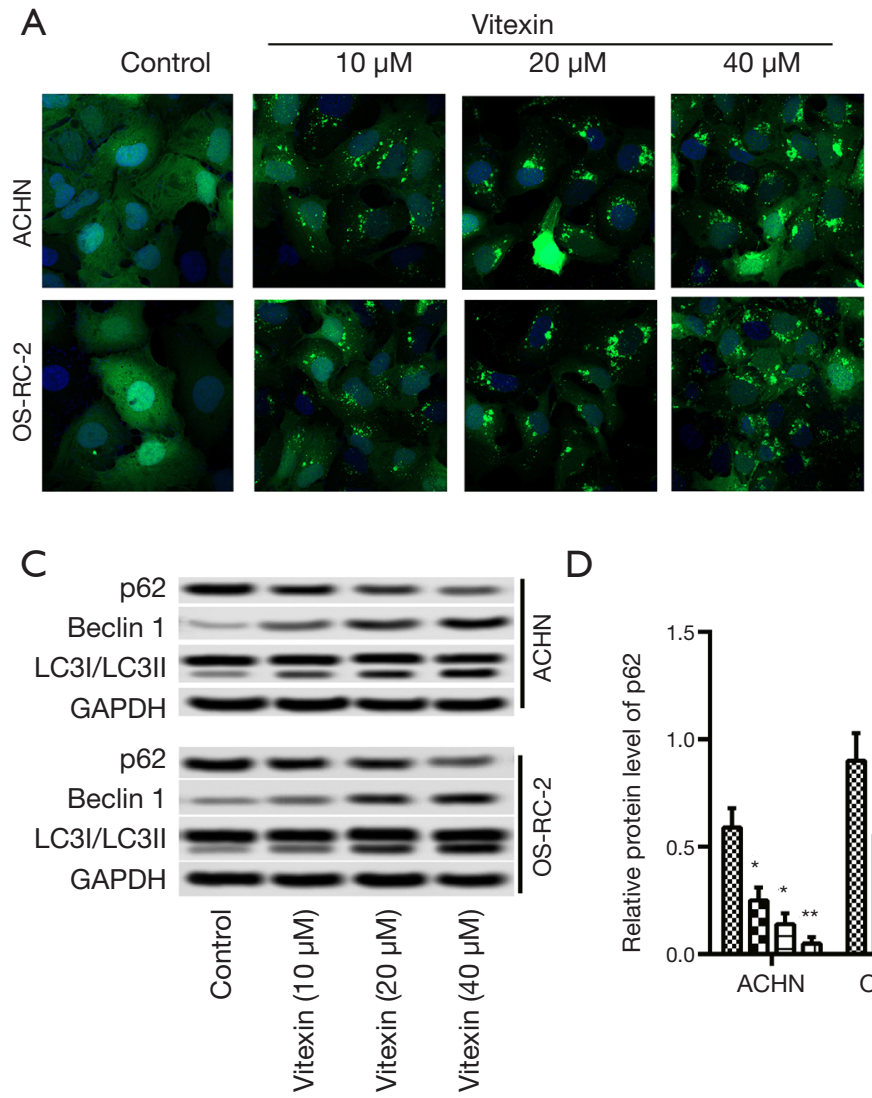

B

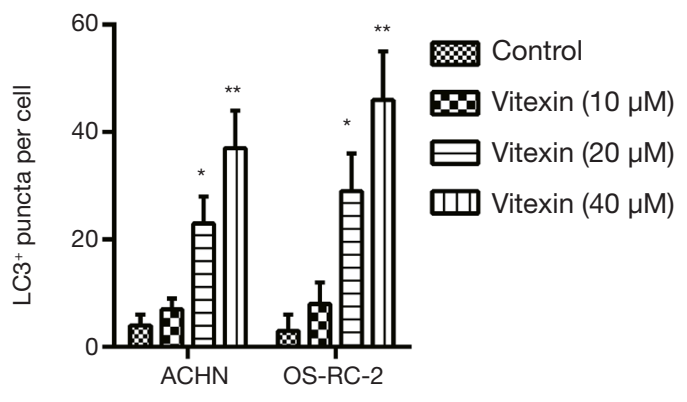

$E$
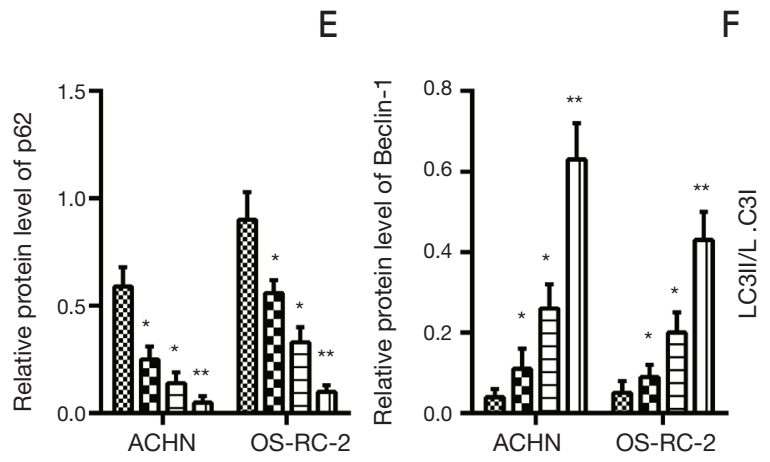

$\mathrm{F}$

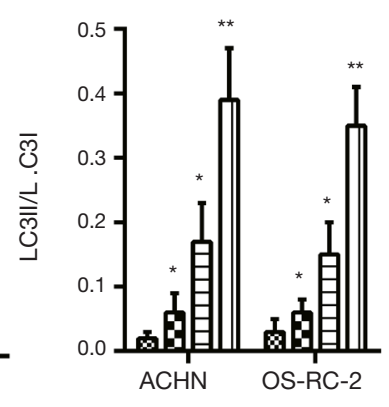

Figure 3 Vitexin induces hyperautophagy of ACHN and OS-RC-2 cells. ACHN and OS-RC-2 cells were treated with vitexin (10, 20, $40 \mu \mathrm{M}$ ) for $24 \mathrm{~h}$. (A) Cell autophagy was evaluated by immunofluorescent assay observed under $400 \times$ scope. (B) LC3 puncta per cell were evaluated with Image Pro Plus. Protein levels of p62, Beclin1, and LC3II/LC3 were assessed by Western blotting (C) and quantized with Image J (D,E,F). ( ${ }^{*}, \mathrm{P}<0.05$ vs. control; ${ }^{* *}, \mathrm{P}<0.01$ vs. control).

of Ki67 (Figure 6C) and Caspase-3 (Figure 6C) in tumor tissue. These results show that vitexin treatment inhibits tumor formation in vitro and in vivo, possibly through regulating AMPK/mTOR, PI3K/AKT/mTOR, and JNK pathways, which was concluded in Figure $6 \mathrm{D}$.

\section{Discussion}

After bladder cancer, RCC is the most common urinary system disease in humans. In China, the incidence of RCC increases each year. Routine radiology helps early diagnosis; however, patients with advanced disease or widespread metastases remain incurable (18). Although surgery and targeted therapy are beneficial, drug resistance limits their application. Therefore, it is important to find new drugs with minimal side effects for the treatment of RCC. This study proposed a new, biologically active compound that inhibited the proliferation of ACHN and OS-RC-2 cells, induced apoptosis and autophagy, and provided a new therapeutic method for clinical treatment of RCC.

Studies show that vitexin promotes apoptosis and autophagy through various signaling pathways, inhibits cell proliferation and migration, and thus exerts antitumor effects (19). Vitexin inhibits the cell cycle and induces apoptosis in glioblastoma cells by inhibiting the AKT/ mTOR pathway (20). It also inhibits the expression of Hsp90, a protein that plays an important role in tumor angiogenesis and metastasis (21). There is evidence that vitexin inhibits proliferation and induces apoptosis of human breast cancer cells (22), as well as significantly inhibiting cell proliferation and inducing apoptosis by modulating levels of p53 and Bcl-2 (23). Other research indicates that 
A

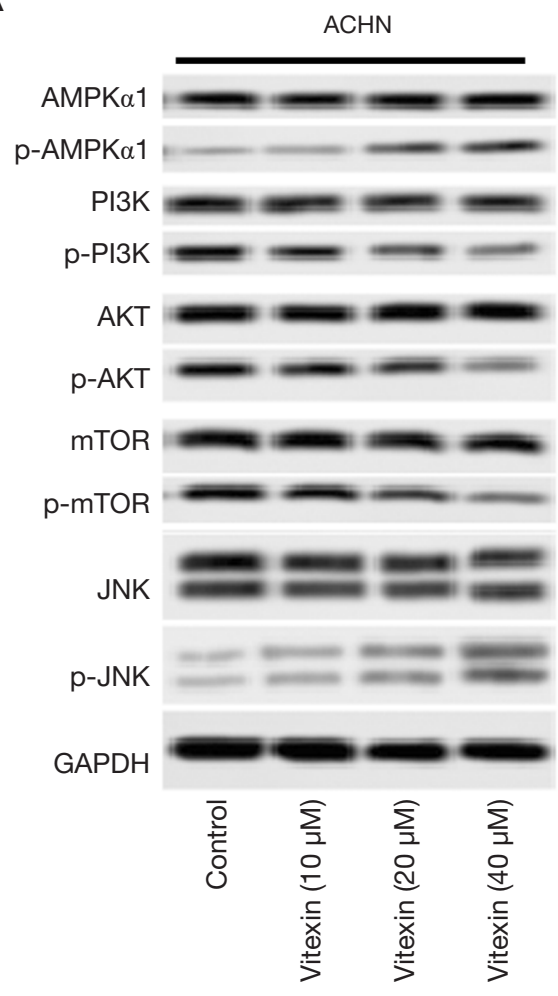

B OS-RC-2
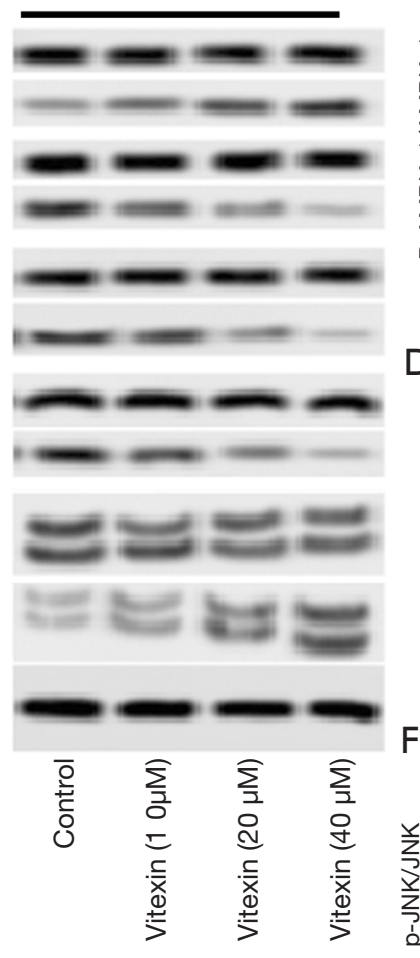

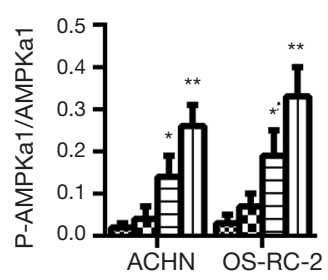

D $\quad E$

C
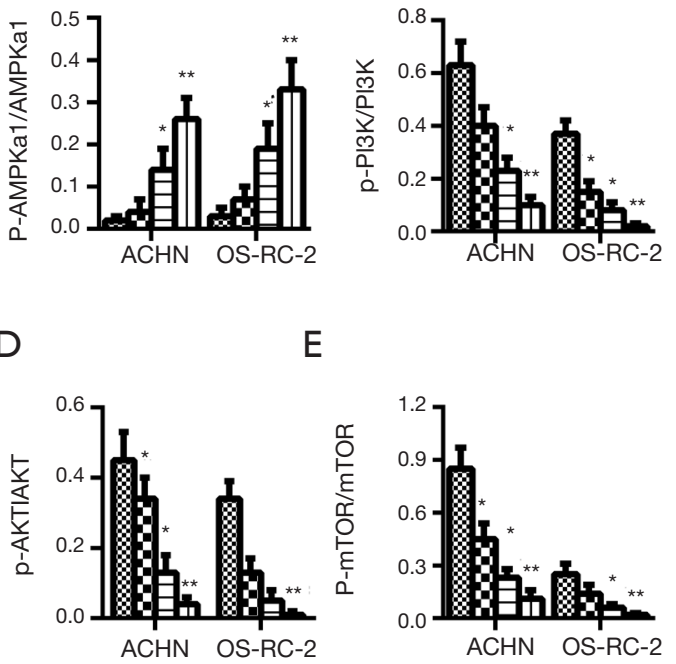

$\mathrm{F}$

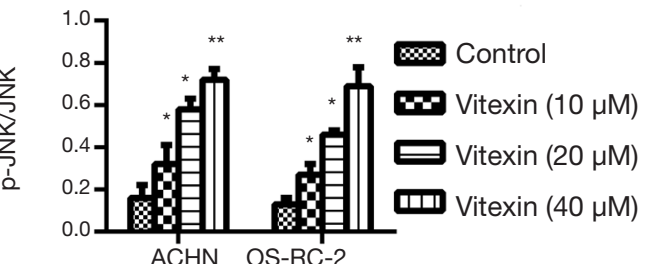

Figure 4 Vitexin dose-dependently regulates AMPK/mTOR, PI3K/AKT/mTOR, and JNK pathways in ACHN and OS-RC-2 cells. ACHN and OS-RC-2 cells were treated with vitexin (10, 20, $40 \mu \mathrm{M}$ ) for $24 \mathrm{~h}$. (A) Protein levels of AMPK/mTOR, PI3K/AKT/mTOR, and JNK pathways were evaluated by Western blotting. Relative protein level of p-AMPKa1/AMPKa1 (B), p-PI3K/PI3K (C), p-AKT/AKT (D), p-mTOR/mTOR (E), and p-JNK/JNK (F) were analyzed with Image J. $\left(^{*}, \mathrm{P}<0.05\right.$ vs. control; **, $\mathrm{P}<0.01$ vs. control). AMPK/mTOR, mammalian target of rapamycin; PI3K/AKT, phosphatidylinositol 3-kinase/activates protein kinase; JNK, c-Jun N-terminal kinase.

vitexin inhibits autophagy (24) of hepatocellular carcinoma and multidrug resistance of colorectal cancer cells (5). In the current study, results show that vitexin inhibited the growth of RCC cells and induced apoptosis and autophagy by regulating the expression of related proteins.

Beclin1, P62 and LC3 are three marker proteins for autophagy. Beclin1 is an autophagy initiation factor that accelerates autophagy (25). Accumulation of LC3 reduces the level of p62, inhibiting tumor growth. Zhang et al. found that thymoquinone promotes the expression of LC3 in 786-O and ACHN cells, thereby inducing autophagy (12). Chai et al. determined the expression of Beclin-1 and LC3II are increased with the overexpression of AIM2 in renal cancer cells (26). Similarity, in the present study, vitexin caused elevation in levels of Beclin1 and LC3 in ACHN and OS-RC-2 cells, while levels of $\mathrm{p} 62$ were decreased. These results indicate that vitexin promotes autophagy in RCC.

The mTOR pathway is the main pathway for autophagy. Abnormal regulation of mTOR promotes tumorigenesis and development (27); therefore, blocking the mTOR pathway may be effective in treating tumors. The mTOR pathway can be regulated by PI3K/AKT and AMPK $(28,29)$. Previous studies have shown that PI3K/AKT and AMPK have opposite effects on the mTOR pathway: inhibition of PI3K/AKT helps to inhibit mTOR pathway, while inactivation of AMPK promotes the mTOR pathway (30,31). Sachan et al. claims that inhibition of PI3K/AKT by Afrocyclamin A blocks the mTOR pathway and promotes 
A

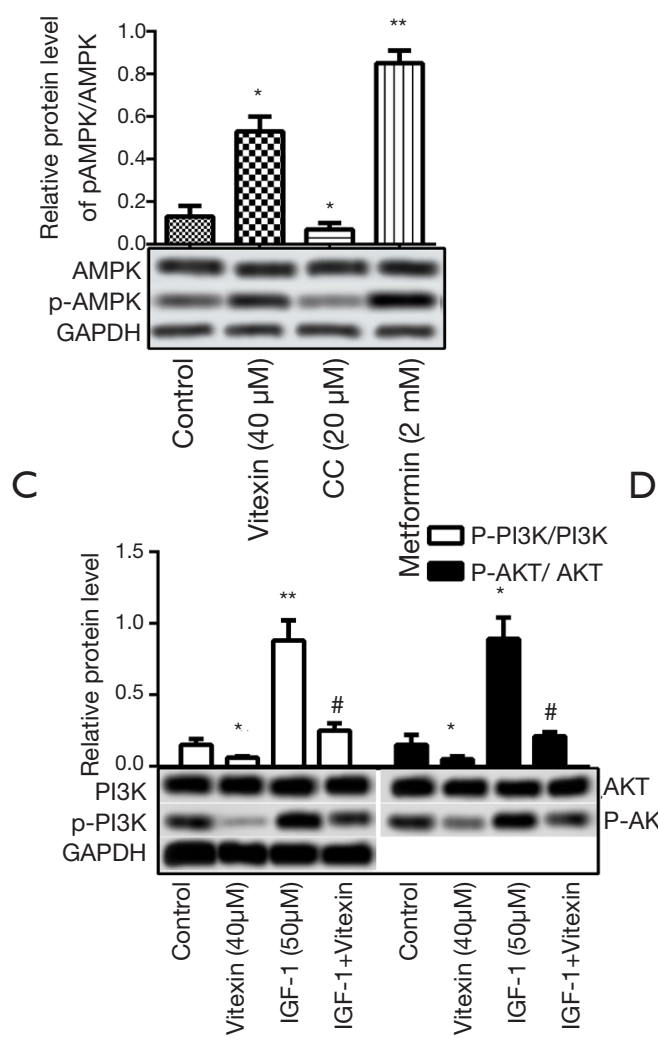

B

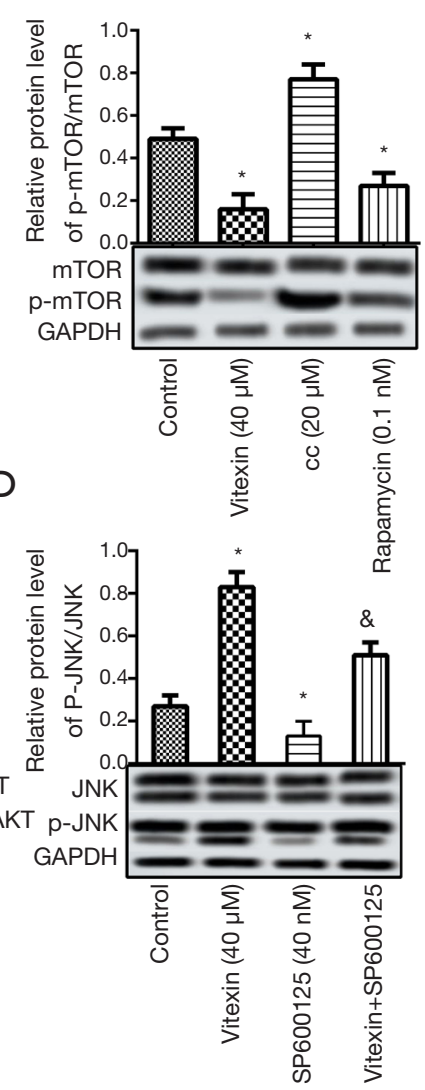

Figure 5 Vitexin inhibits the growth of OS-RC-2 cells and induces apoptosis and hyperautophagy through AMPK/mTOR, PI3K/AKT/ mTOR, and JNK pathways. OS-RC-2 cells were treated with vitexin $(40 \mu M)$, AMPK inhibitor [Compound C (CC, $20 \mu M)]$, metformin (2 mM), IGF-1 (50 $\mu \mathrm{M})$ and/or SP600125 (40 nM) as indicated. Protein levels of p-AMPK/AMPK (A), p-mTOR/mTOR (B), p-PI3K/PI3K \& pAKT/AKT $(\mathrm{C})$, and p-JNK/JNK (D) were monitored by western blotting and analyzed with Image $\mathrm{J}$. ( ${ }^{*}, \mathrm{P}<0.05$ vs. control; **, $\mathrm{P}<0.01$ vs. control; \#, $\mathrm{P}<0.05$ vs. IGF-1; \&, $\mathrm{P}<0.05$ vs. SP600125). AMPK/mTOR, mammalian target of rapamycin; PI3K/AKT, phosphatidylinositol 3-kinase/activates protein kinase; JNK, c-Jun N-terminal kinase.

apoptosis and autophagy in prostate cancer cells (32). In addition, Li et al. showed that ginsenoside metabolite $\mathrm{K}$-induced AMPK activation inhibits phosphorylation of mTOR, thereby inducing apoptosis and autophagy in lung cancer cells (29). In this study, the level of p-AMPK was significantly increased in ACHN and OS-RC-2 cells treated with vitexin. Enhanced p-AMPK level inhibited the activation of mTOR. Vitexin also reduced the phosphorylation of PI3K and AKT in ACHN and OSRC-2 cells, and increased the phosphorylation level of JNK. Inactivation of the PI3K/AKT signaling pathway further inhibited phosphorylation of mTOR, whereas activation of the JNK pathway accelerated autophagy. These results indicate that vitexin can play an important role in $\mathrm{ACHN}$ and OS-RC-2 cells by upregulating the AMPK/mTOR and JNK pathways, and downregulating the PI3K/AKT/mTOR pathway.

In conclusion, this study clarified the role of vitexin in RCC and its corresponding molecular mechanism, both in vitro and in vivo. The study demonstrated that in vitro vitexin $(10,20$ and $40 \mu \mathrm{M})$ can significantly inhibit the growth of ACHN and OS-RC-2 cells and induce apoptosis and hyperautophagy in a dose-dependent manner by upregulating the AMPK/mTOR and JNK pathways and downregulating PI3K/AKT/mTOR pathways. In addition, vitexin can inhibit tumor formation in vivo. Therefore, 
A

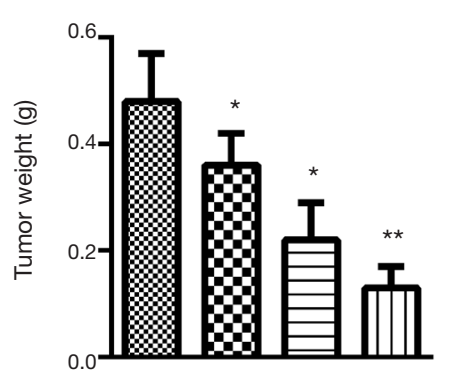

C
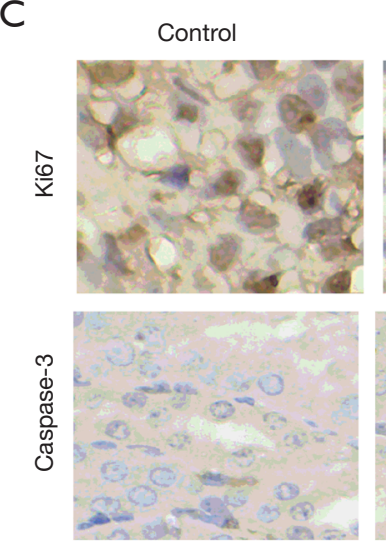

D

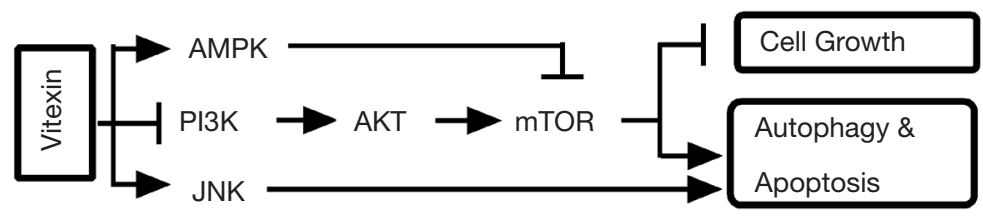

B
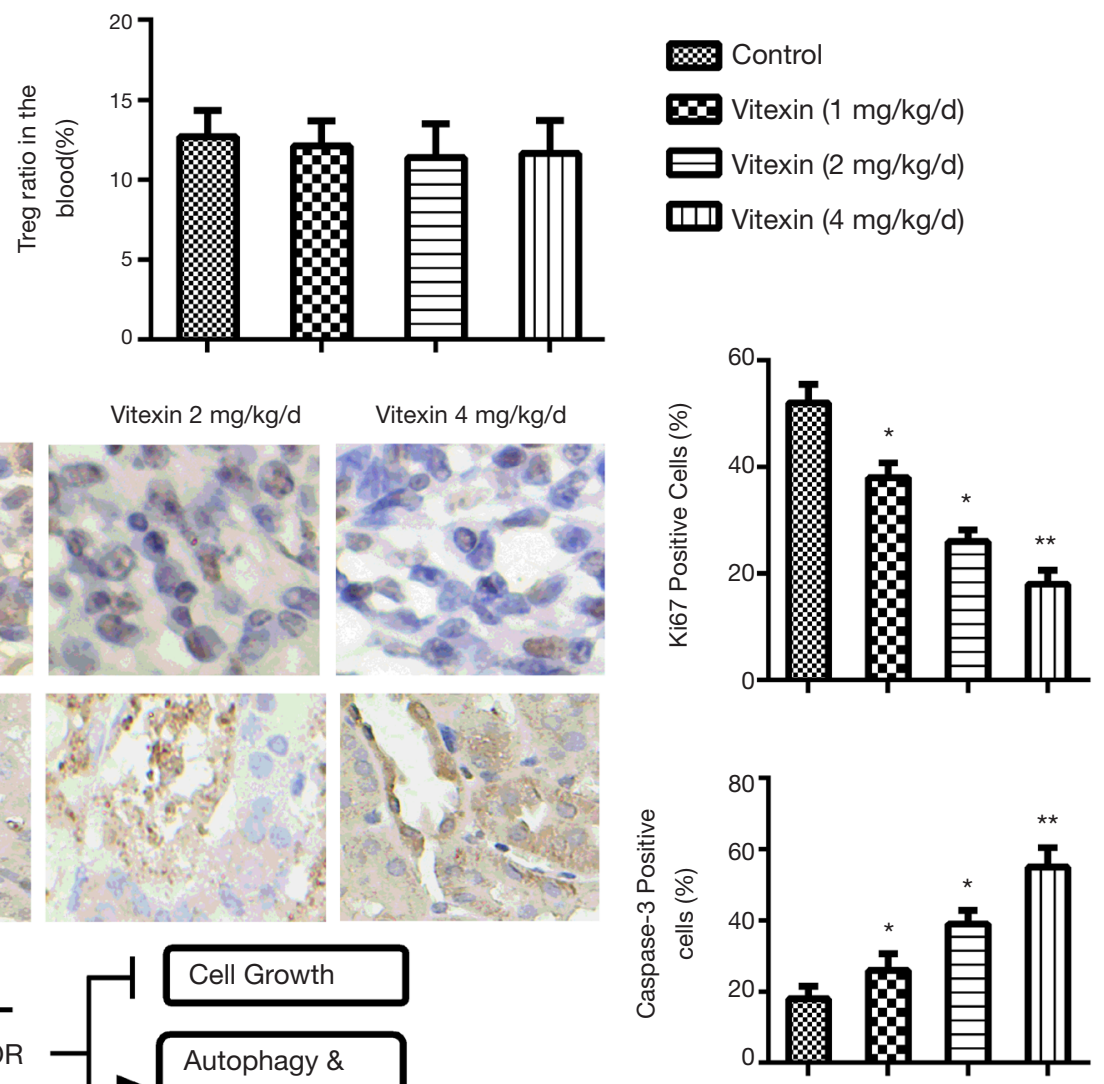

Figure 6 Vitexin inhibits tumor formation in vivo. The mice were divided into four groups $(\mathrm{n}=10)$ and treated for 25 days as: control group (saline); vitexin $1 \mathrm{mg} / \mathrm{kg} / \mathrm{d}$; vitexin $2 \mathrm{mg} / \mathrm{kg} / \mathrm{d}$; and vitexin $4 \mathrm{mg} / \mathrm{kg} / \mathrm{d}$. (A) Tumors were weighted. (B) The ratio of Tregs in the peripheral blood. (C) The expression of Ki67 and Caspase-3 in tumor tissues measured by IHC and observed under 200x scope. (D) Mechanism of action for vitexin validated in this research. $\left({ }^{*}, \mathrm{P}<0.05\right.$ vs. control; ${ }^{* *}, \mathrm{P}<0.01$ vs. control).

vitexin may be a promising drug for the treatment of RCC.

\section{Acknowledgments}

Funding: This work was supported by the Key R \& D plan of Shandong Province (special public welfare project) (No.: 2019GSF108172); key R \& D plan of Jining (Science and technology to promote the transformation of new and old kinetic energy) (No.: 2019SMNS003); Shandong Province TCM science and technology development plan (No.:2019749); and Jining TCM science and technology project plan (No.: ZYY015018).

\section{Footnote}

Reporting Checklist: The authors have completed the ARRIVE reporting checklist. Available at http://dx.doi. org/10.21037/tau-20-1094

Data Sharing Statement: Available at http://dx.doi. org/10.21037/tau-20-1094

Conflicts of Interest: All authors have completed the ICMJE uniform disclosure form (available at http://dx.doi. org/10.21037/tau-20-1094). The authors have no conflicts of interest to declare. 
Ethical Statement: The authors are accountable for all aspects of the work in ensuring that questions related to the accuracy or integrity of any part of the work are appropriately investigated and resolved. All animal experiments were performed in accordance with the National Institutes of Health (NIH) Guide for the Care and Use of Laboratory Animals and were approved by Guizhou Medical University.

Open Access Statement: This is an Open Access article distributed in accordance with the Creative Commons Attribution-NonCommercial-NoDerivs 4.0 International License (CC BY-NC-ND 4.0), which permits the noncommercial replication and distribution of the article with the strict proviso that no changes or edits are made and the original work is properly cited (including links to both the formal publication through the relevant DOI and the license). See: https://creativecommons.org/licenses/by-nc-nd/4.0/.

\section{References}

1. Ferlay J, Soerjomataram I, Dikshit R, et al. Cancer incidence and mortality worldwide: sources, methods and major patterns in GLOBOCAN 2012. Int J Cancer 2015;136:E359-86.

2. Zhong J, Wah TM. Renal ablation: current management strategies and controversies. Chin Clin Oncol 2019;8:63.

3. Yan WU, Yang H, Chen S, et al. Determination of total flavonoids and vitexin in the resin extracts of Fenugreek seeds. West China Journal of Pharmaceutical Sciences 2010;25:478-80.

4. Nurdiana S, Goh YM, Ahmad H, et al. Changes in pancreatic histology, insulin secretion and oxidative status in diabetic rats following treatment with Ficus deltoidea and vitexin. BMC Complement Altern Med 2017;17:290.

5. Bhardwaj M, Cho HJ, Paul S, et al. Vitexin induces apoptosis by suppressing autophagy in multi-drug resistant colorectal cancer cells. Oncotarget 2017;9:3278-91.

6. Wang Z, Cai J, Fu Q, et al. Anti-Inflammatory Activities of Compounds Isolated from the Rhizome of Anemarrhena asphodeloides. Molecules 2018;23:2631.

7. Choo CY, Sulong NY, Man F, et al. Vitexin and isovitexin from the Leaves of Ficus deltoidea with in-vivo $\alpha$-glucosidase inhibition. J Ethnopharmacol 2012;142:776-81.

8. Vasant OK, Vijay BG, Virbhadrappa SR, et al. Antihypertensive and Diuretic Effects of the Aqueous Extract of Colocasia esculenta Linn. Leaves in Experimental Paradigms. Iran J Pharm Res 2012;11:621-34.
9. Qi HY, Qu XJ, Liu J, et al. Bufalin induces protective autophagy by Cbl-b regulating mTOR and ERK signaling pathways in gastric cancer cells. Cell Biol Int 2019;43:33-43.

10. Zhou J, Hu H, Long J, et al. Vitexin 6, a novel lignan, induces autophagy and apoptosis by activating the Jun $\mathrm{N}$-terminal kinase pathway. Anticancer Drugs 2013;24:928-36.

11. Zhu H, Wang J, Shao Y, et al. Catalpol may improve axonal growth via regulating miR-124 regulated PI3K/ AKT/mTOR pathway in neurons after ischemia. Ann Transl Med 2019;7:306.

12. Wang Q, Shen B, Qin X, et al. Akt/mTOR and AMPK signaling pathways are responsible for liver $\mathrm{X}$ receptor agonist GW3965-enhanced gefitinib sensitivity in nonsmall cell lung cancer cell lines. Transl Cancer Res 2019;8:66-76.

13. Tavares MR, Pavan ICB, Amaral CL, et al. The S6K protein family in health and disease. Life Sciences 2015;131:1-10.

14. Chen $Y$, Wang M, Zhang T, et al. Autophagic effects and mechanisms of silver nanoparticles in renal cells under low dose exposure. Ecotoxicol Environ Saf 2018;166:71-7.

15. Liu N, Wang KS, Qi M, et al. Vitexin compound 1, a novel extraction from a Chinese herb, suppresses melanoma cell growth through DNA damage by increasing ROS levels. J Exp Clin Cancer Res 2018;37:269.

16. Li JH, Zhang L, Feng $\mathrm{Y}$, et al. Bortezomib Inhibits Extranodal Natural Killer/T Cell Lymphoma, Nasal Type by Targeting NF-кB Signaling Pathway. Sichuan Da Xue Xue Bao Yi Xue Ban 2019;50:311-6.

17. Yan-xia W, Chun-qiang Z, Fei H. Effects of HOXA1 Gene Antisense Oligonucleotides on Growth of Esophageal Cancer Cells. Sichuan Da Xue Xue Bao Yi Xue Ban 2020;51:24-9.

18. Lin H, Huang B, Wang H, et al. MTHFD2 Overexpression Predicts Poor Prognosis in Renal Cell Carcinoma and is Associated with Cell Proliferation and Vimentin-Modulated Migration and Invasion. Cell Physiol Biochem 2018;51:991-1000.

19. Bhardwaj M, Paul S, Jakhar R, et al. Vitexin confers HSF1 mediated autophagic cell death by activating JNK and ApoL1 in colorectal carcinoma cells. Oncotarget 2017;8:112426-41.

20. Zhang G, Li D, Chen H, et al. Vitexin induces G2/ $\mathrm{M}$-phase arrest and apoptosis via Akt/mTOR signaling pathway in human glioblastoma cells. Mol Med Rep 2018; 17:4599-604.

21. Sharma N, Akhtar S, Jamal QMS, et al. Elucidation 
of Antiangiogenic Potential of Vitexin Obtained from Cucumis sativus Targeting Hsp90 Protein: A Novel Multipathway Targeted Approach to Restrain Angiogenic Phenomena. Med Chem 2017;13:282-91.

22. Czemplik M, Mierziak J, Szopa J, et al. Flavonoid C-glucosides Derived from Flax Straw Extracts Reduce Human Breast Cancer Cell Growth In vitro and Induce Apoptosis. Front Pharmacol 2016;7:282.

23. An F, Wang S, Tian Q, et al. Effects of orientin and vitexin from Trollius chinensis on the growth and apoptosis of esophageal cancer EC-109 cells. Oncol Lett 2015;10:2627-33.

24. He JD, Wang Z, Li SP, et al. Vitexin suppresses autophagy to induce apoptosis in hepatocellular carcinoma via activation of the JNK signaling pathway. Oncotarget 2016;7:84520.

25. Aita VM, Liang XH, Murty VV, et al. Cloning and genomic organization of beclin 1 , a candidate tumor suppressor gene on chromosome 17q21. Genomics 1999;59:59-65.

26. Chai D, Shan H, Wang G, et al. AIM2 is a potential therapeutic target in human renal carcinoma and suppresses its invasion and metastasis via enhancing autophagy induction. Experimental Cell Research 2018;370:561-70.

27. Way TD, Tsai SJ, Wang CM, et al. Chemical constituents

Cite this article as: Li Y, Sun Q, Li H, Yang B, Wang M. Vitexin suppresses renal cell carcinoma by regulating mTOR pathways. Transl Androl Urol 2020;9(4):1700-1711. doi: 10.21037/ tau-20-1094 of Rhododendron formosanum show pronounced growth inhibitory effect on non-small-cell lung carcinoma cells. J Agric Food Chem 2014;62:875-84.

28. Corti F, Nichetti F, Raimondi A, et al. Targeting the PI3K/ AKT/mTOR pathway in biliary tract cancers: A review of current evidences and future perspectives. Cancer Treat Rev 2019;72:45-55.

29. Li C, Dong Y, Wang L, et al. Ginsenoside metabolite compound $\mathrm{K}$ induces apoptosis and autophagy in nonsmall cell lung cancer cells via AMPK-mTOR and JNK pathways. Biochem Cell Biol 2019;97:406-14.

30. Sun B, Ou H, Ren F, et al. Propofol inhibited autophagy through $\mathrm{Ca}(2+) / \mathrm{CaMKK} \beta / \mathrm{AMPK} / \mathrm{mTOR}$ pathway in OGD/R-induced neuron injury. Mol Med 2018;24:58.

31. Li B, Xi P, Wang Z, et al. PI3K/Akt/mTOR signaling pathway participates in Streptococcus uberis-induced inflammation in mammary epithelial cells in concert with the classical TLRs/NF-кB pathway. Vet Microbiol 2018;227:103-11.

32. Sachan R, Kundu A, Jeon Y, et al. Afrocyclamin A, a triterpene saponin, induces apoptosis and autophagic cell death via the PI3K/Akt/mTOR pathway in human prostate cancer cells. Phytomedicine 2018;51:139-50.

(English Language Editors: J. Brown and J. Gray) 